\title{
Nonsuicidal Self-Injury among Korean Young Adults: A Validation of the Korean Version of the Inventory of Statements about Self-Injury
}

\author{
Soyeon Kim, Yoongyeong Kim, and Ji-Won Hur ${ }^{\bowtie}$ \\ Department of Psychology, Chung-Ang University, Seoul, Republic of Korea
}

Objective The purpose of this study was to validate and verify the Korean version of the Inventory of Statements about Self-Injury (ISAS) to better understand the psychopathological characteristics of nonsuicidal self-injury (NSSI) among young adults.

Methods A total of 539 Korean adults (age: 19 to 30 years; 343 participants with a history of NSSI) completed a self-report online survey regarding NSSI validation research. The test-retest reliability, internal consistency, concurrent validity, and factor analysis of the ISAS behavioral scales and the ISAS functional scales were examined. The factor structure and construct validity were evaluated by performing exploratory factor analysis (EFA) and a confirmatory factor analysis (CFA).

Results The results demonstrated the good internal consistency and temporal stability of the Korean version of the ISAS. The EFA revealed that the NSSI functions exhibited a two-factor structure: intrapersonal functions and interpersonal functions. The CFA also confirmed that the model fitness indicators of the 2 -factor structure were appropriate. In addition, the ISAS functional scales were related to increased suicidal ideations and decreased resilience in participants with a history of NSSI.

Conclusion The current findings suggest that the Korean version of the ISAS is a robust measure of NSSI behaviors. Further research is needed to investigate the causal relationship between clinical symptoms, given the significant correlation between self-reported NSSI and suicidal ideation and decreased resilience.

Psychiatry Investig 2019;16(4):270-278

Key Words Nonsuicidal self-injury, Self-injurious behavior, Validation, Suicide, Resilience.

\section{INTRODUCTION}

Nonsuicidal self-injury (NSSI) is deliberate and repetitive self-harming behaviors aimed at the destruction of one's own body tissues or organs. NSSI manifests as a wide variety of behavioral patterns. Typical NSSI behaviors include cutting or carving the skin, burning the skin or deliberately fracturing one's bones. Sometimes self-injurious behaviors are easily hidden or passed off as accidents, ${ }^{1}$ such as lip biting, hitting the edge of furniture or falling. Concealed NSSI behaviors may interfere with accurate estimates of NSSI prevalence, which is a highly prevalent pathological symptom among both clinical

\footnotetext{
Received: April 6, 2018 Revised: July 16, 2018

Accepted: January 23, 2019

$\bowtie$ Correspondence: Ji-Won Hur, PhD

Department of Psychology, Chung-Ang University, 84 Heukseok-ro, Dongjak-gu, Seoul 06974, Republic of Korea

Tel: +82-2-820-5744, Fax: +82-2-816-5124, E-mail: neuropsy.hur@gmail.com

(a) This is an Open Access article distributed under the terms of the Creative Commons Attribution Non-Commercial License (https://creativecommons.org/licenses/bync/4.0) which permits unrestricted non-commercial use, distribution, and reproduction in any medium, provided the original work is properly cited.
}

and community samples. For instance, the estimated prevalence of NSSI is $13-46.5 \%$ for adolescents and $11.7-38.9 \%$ for college students. ${ }^{2-7}$ In a recent study of female middle school students in South Korea, approximately one-fifth of the research participants reported NSSI experiences. ${ }^{8}$ Although individuals who engaged in NSSI may argue that their actions are carried out with no intent to commit suicide, this voluntary and destructive attempt leads to suicidal behaviors, including suicidal ideation and suicide attempts., ${ }^{9,10}$

NSSI behaviors occur and are perpetuated in relation to a series of functions: intrapersonal functions (i.e., escaping from unwanted ideations or feelings or leading to desired feelings or stimulation) and interpersonal functions (i.e., leading to peer bonding or escaping from undesired interpersonal stimuli). ${ }^{11-14} \mathrm{~A}$ recent meta-analysis revealed that intrapersonal functions aimed at modulating one's internal environment were more common (used by $66-81 \%$ of individuals who engage in NSSI) than interpersonal functions aimed at influencing one's external environment. ${ }^{15}$ In Korea, this pattern appears even more prominent, and approximately $90 \%$ of individuals 
who engage in NSSI report that intrapersonal functions facilitate self-injurious behaviors. ${ }^{16}$

To explore various functions that have a great influence on the onset or maintenance of NSSI behaviors, the Inventory of Statements about Self-Injury (ISAS) was developed to cover more extensive functions that drive NSSI and are related to sensation seeking, coping with dissociative experiences or suicidal ideation, and establishing personal boundaries. ${ }^{17}$ Several measures have been developed to examine NSSI characteristics, such as the Deliberate Self-Harm Inventory (DSHI) ${ }^{18}$ Suicide Attempt Self-Injury Interview (SASII), ${ }^{19}$ and Functional Assessment of Self-Mutilation (FASM); ${ }^{20}$ however, these NSSI scales have limitations in that they were not originally designed with the functions of NSSI behaviors in mind. ${ }^{21}$ Hereupon, Klonsky and Glenn ${ }^{17}$ developed the ISAS with a sample of 235 young adults in order to comprehensively assess both the function and frequency of self-injurious behaviors. The original version of the ISAS explores 13 functions of NSSI (i.e., affect regulation, avoiding suicide, marking distress, selfpunishment, anti-dissociation, interpersonal boundaries, interpersonal influence, revenge, sensation seeking, peer bonding, toughness, autonomy, and self-care) along with the frequency of NSSI, which is closely associated with higher levels of suicidal ideation. ${ }^{10,22}$ Because of its abundance of information on both the phenomenology and functions of NSSI, the ISAS has been regarded as a promising measurement for case conceptualization and treatment planning in clinical settings. ${ }^{17}$ Given the virtues of the measure, the has been translated to and validated in three languages (Turkish, Mexican Spanish, and Swedish). ${ }^{12,23,24}$

The current study was conducted to confirm the factor structure, internal consistency, test-retest reliability, and concurrent validity of the Korean version of the ISAS. We also explored the link between NSSI patterns with suicidal ideation and resilience, which are closely related to self-destructive behavior. ${ }^{25,26}$ Thus, we assumed that the Korean version of the ISAS had adequate psychometric features and provided clinical insights into mental health problems in South Korean youth.

\section{METHODS}

\section{Participants and procedure}

Consistent with the validation process for the original versions of the NSSI, which examined young adults with the most frequent levels of depression, ${ }^{17,27}$ the subjects consisted of Korean adults aged 19 to 30 years who were recruited through internet advertisements posted on websites for different groups (e.g., online community groups, online hobby groups, and online NSSI forums). The anticipated sample size was 347 based on a sample size calculator; ${ }^{28}$ and a $5.26 \%$ confidence interval and 95\% confidence level were used, which is consistent with a previous study. ${ }^{12}$ In Korea, the population aged 19 to 30 years numbers 7,731,010 according to Statistics Korea 2016. ${ }^{29}$ The exclusion criteria were as follows:

1) age younger than 19 or older than 30 years, 2) display of comprehension problems, and 3) lack of motivation or cooperation in responding to the questionnaires.

Eligible participants were asked to independently complete the online survey via the internet. Of the 757 participants, 74 respondents were excluded due to the omission of the ISAS and 144 respondents were excluded due to the omission of other scales. The final sample consisted of 196 controls (control group: never engaged in NSSI during their lifetime) and 343 NSSI participants (NSSI group: engaged in NSSI more than once during their lifetime ${ }^{12,17,30}$ ).

The study protocol was approved by the Institutional Review Board of Chung-Ang University (IRB No. 1041078-2017 06-BRSB-127-0C), and we obtained written informed consent from all participants in this study.

\section{Instruments}

\section{Inventory of Statements about Self-Injury (ISAS)}

NSSI behaviors and functions were assessed using the Korean version of the ISAS (Supplementary Material 1 in the online-only Data Supplement). ${ }^{17,22}$ The first section of the ISAS measures the lifetime frequency of 12 NSSI behaviors (e.g., burning, cutting, and carving) performed intentionally without suicidal intent. Here, the frequency distribution of each self-injurious behavior was defined according to previous research, and the first section was scored as 0 (none), 1 (few), 2 (moderate), or 3 (common) based on the frequency distribution of each self-injurious behavior to facilitate a statistical analysis. ${ }^{12}$ The second section of the ISAS consisted of 39 items that assess 13 NSSI functions, such as affect regulation and autonomy, ${ }^{22}$ which diverged into 2 superordinate categories: intrapersonal function and interpersonal function. The second section was rated on a scale from 0 (not at all relevant) to 2 (very relevant) according to the respondent's experience of NSSI functions (Cronbach's alpha for the internal consistency of sections 1 and $2=0.84$ and 0.71 , respectively). ${ }^{22}$

The Korean version of the ISAS (Supplementary Material 1 in the online-only Data Supplement) was developed using the back-translation method, ${ }^{31}$ which is recommended for use in cross-cultural research. ${ }^{32}$ First, the ISAS was translated into Korean by two native Koreans who were fluent in English. Then, the Korean version of the ISAS was back-translated into English by another bilingual translator from the research team to confirm whether the translation matched the wording of the original scale. Finally, the Korean version was compared 
with the original version by an English-speaking consensus committee of clinicians. When discrepancies occurred between the two versions, individual items were subjected to additional rounds of back-translation until they were deemed satisfactory.

\section{Functional Assessment of Self-Mutilation (FASM)}

The FASM was developed by Lloyd et al. ${ }^{20}$ to measure the frequency and motives of self-mutilation. The first part of the FASM assesses self-mutilation methods, frequencies and behaviors. Self-mutilation motives are measured in the second part, which consists of 22 items. The reported internal consistency was 0.65 to $0.66 .{ }^{20}$ In Korea, the FASM was adapted and validated by Kwon and Kwon (Cronbach's alpha=0.80). ${ }^{30}$

\section{Beck Scale for Suicidal Ideation (BSI)}

Because suicidal ideation is one of the clear predictors of suicidal attempts, further research is needed on the relevance of suicidal ideation to NSSI behaviors. ${ }^{2}$ The BSI consists of 19 items developed for clinical interviews. ${ }^{33}$ Suicidal ideation has 3 dimensions, including active suicidal desire, specific plans for suicide and passive suicidal desire. The BSI is scored using a 3-point Likert scale, and the total score ranges from 0 to 38 (Cronbach's alpha $=0.89$ ). ${ }^{33}$ Generally, a total score of 6 or more is considered clinically significant. ${ }^{34}$ In South Korea, Shin et al..$^{35}$ modified the BSI into a self-report scale (Cronbach's alpha $=0.81) .{ }^{35}$

\section{Korean Resilience Questionnaire-53 (KRQ-53)}

Resilience is defined as the ability to bounce back or recover from stress, adapt to stressful situations, and function above the norm despite untoward stress and adversity. ${ }^{36}$ The Korean version of the Resilience Questionnaire (KRQ-53; Cronbach's alpha ranged from 0.79 to 0.89 ) was adapted from the questionnaire of the Resilience Quotient Test (RQT). ${ }^{37,38}$ The KRQ53 consists of 9 subscales and diverges into 3 superordinate categories including control (causal analysis, emotional regulation, and impulse control), interpersonality (communication, sympathy, and relationship), and positivity (optimism, daily satisfaction, and appreciation). After informed consent was obtained, the ISAS, FASM, BSI and KRQ-53 were administered.

\section{Statistical analysis}

All data were assessed using a SPSS 18 for Windows (SPSS Inc., Chicago, IL, USA) spreadsheet. In terms of reliability, the test-retest reliability and internal consistency were investigated. To examine the retest reliability, 70 individuals were randomly selected from the total sample to retake the Korean version of the ISAS 4 weeks after the first administration. Thirty- five subjects completed the 4-week retest and were included in the test-retest analysis.

In addition, Mplus and AMOS 23 were used to perform exploratory factor analysis (EFA) and confirmatory factor analysis (CFA), respectively. EFA was conducted first to identify the underlying structure of the ISAS by adopting the maximum likelihood extraction method with an oblique promax rotation. ${ }^{39}$ Consistent with a previous study, ${ }^{11}$ the following considerations were integrated for retaining the number of factors: inspection of the scree plot based on the redundancy of factors (identify the number of factors above the breaking point or "elbow"), the conceptual interpretability of factors, the Kaiser criterion (factors with eigenvalues greater than 1) and variance explained for each factor. Then, the CFA was performed to verify the factor structure of the ISAS scale items. ${ }^{12,17}$

Regarding the CFA model, we followed the recommendations of $\mathrm{Hu}$ and Bentler ${ }^{40}$ and other researchers regarding the goodness-of-fit indices: root mean square error of approximation (RMSEA) values $\leq 0.05$ indicated a good fit, values between 0.05 and 0.08 indicated an adequate fit, and values between 0.08 and 0.10 indicated a mediocre fit. ${ }^{41-43}$ Additionally, a model was considered adequate if the comparative fit index (CFI) was above $0.90 ;{ }^{44}$ and a root mean square residual (RMR) value $<0.05$ was considered to indicate a good fit. ${ }^{45}$ Correlations between the Korean version of the ISAS and other scales were also analyzed to further investigate the psychopathological characteristics of NSSI among Korean young adults.

\section{RESULTS}

\section{Demographic and clinical characteristics}

Of the 539 participants, $66.2 \%(n=357)$ were female, and $0.7 \%(n=4)$ were "sex unspecified." A total of $48 \%(n=262)$ of the participants were aged 19 to 22 years, $40.2 \%(n=217)$ were aged 23 to 26 years, and $11.2 \%(n=60)$ were aged 27 to 30 years. Regarding education level, the participants reported the following: $0.2 \%(n=1)$ had "not completed high school," $3.3 \%(n=18)$, were a "high school graduate," 67.7\% ( $n=365)$ were "in university," $20.4 \%(n=110)$ were a "university graduate," and $8.3 \%(n=45)$ were "beyond graduate school." In terms of self-reported socioeconomic status, the participants reported the following: $7.1 \%(n=38)$ were low, $22.3 \%(n=20)$ were low-medium, $40.3 \%(n=217)$ were medium, $26 \%(n=140)$ were medium-high, and $4.5 \%(\mathrm{n}=24)$ were high.

\section{Section 1 of the ISAS (ISAS behavioral scales)}

\section{Reliability analysis}

The test-retest reliability and internal consistency were an- 
Table 1. Correlations between the overall score and each item in the ISAS section $1(\mathrm{~N}=539)$

\begin{tabular}{lc}
\hline \multicolumn{1}{c}{ ISAS each behavior } & ISAS section 1 overall \\
\hline Banging or hitting self & $0.77^{*}$ \\
Biting & $0.75^{*}$ \\
Burning & $0.44^{*}$ \\
Carving & $0.40^{*}$ \\
Cutting & $0.56^{*}$ \\
Interfering with wound healing & $0.63^{*}$ \\
Pinching & $0.72^{*}$ \\
Pulling hair & $0.64^{*}$ \\
Rubbing skin against rough surface & $0.58^{*}$ \\
Severe scratching & $0.60^{*}$ \\
Sticking self with needles & $0.61^{*}$ \\
Swallowing dangerous substances & $0.50^{*}$ \\
Else & $0.44^{*}$ \\
\hline
\end{tabular}

${ }^{*} \mathrm{p}<0.05$. ISAS: Inventory of Statements about Self-Injury

alyzed to verify the reliability of the Korean version of the ISAS. The internal consistency of ISAS section 1 was excellent (Cronbach's alpha $=0.85, \mathrm{n}=539$ ). Pearson correlations between the overall score and each item ranged from 0.40 (carving) to 0.77 (banging or hitting oneself), and the median was 0.60 (severe scratching) (all $\mathrm{p}<0.001)$ (Table 1).

The four-week test-retest reliability of section 1 was examined using data from 35 participants who were randomly selected from among all participants. The test-retest correlation of the overall score was significantly high $(\mathrm{r}=0.90, \mathrm{p}<0.001)$. The test-retest correlations for each behavior varied from 0.50 (sticking oneself with needles) to 0.94 (cutting), with a median of 0.74 (pinching). All correlations were positive and statistically significant $(\mathrm{p}<0.001)$.

\section{Validity analysis}

Pearson correlations were calculated between the ISAS behavioral scales and the behavioral frequency of the NSSI measured by the FASM (item "A") to investigate the concurrent validity. The correlation between the 2 measurements was good $(r=0.74, p<0.001)$ (Table 2). All correlations between the frequencies of NSSI behavior measured by the ISAS and FASM were significant and ranged from 0.17 (carving) to 0.59 (biting), with a median of 0.41 (interfering with wound healing) $(\mathrm{p}<0.001)$.

\section{Section 2 of the ISAS (ISAS functional scales)}

\section{Reliability analysis}

Cronbach's alpha was 0.92 for the Korean version of ISAS section 2 , indicating excellent internal consistency reliability.
Table 2. Correlations between the Inventory of the FASM and the ISAS section 1 (overall score and each item) ( $\mathrm{N}=539)$

\begin{tabular}{ll}
\hline \multicolumn{1}{c}{ ISAS each behavior } & FASM \\
\hline ISAS section 1 overall & $0.74^{*}$ \\
Banging or hitting self & $0.59^{*}$ \\
Biting & $0.59^{*}$ \\
Burning & $0.35^{*}$ \\
Carving & $0.17^{*}$ \\
Cutting & $0.59^{*}$ \\
Interfering with wound healing & $0.42^{*}$ \\
Pinching & $0.44^{*}$ \\
Pulling hai & $0.41^{*}$ \\
Rubbing skin against rough surface & $0.34^{*}$ \\
Severe scratching & $0.50^{*}$ \\
Sticking self with needles & $0.43^{*}$ \\
Swallowing dangerous substances & $0.34^{*}$ \\
Else & $0.34^{*}$ \\
\hline
\end{tabular}

${ }^{*} \mathrm{p}<0.05$. ISAS: Inventory of Statements about Self-Injury, FASM: Functional Assessment of Self-Mutilation

The range was 0.24 (item 34) to 0.70 (item 29), and the median was 0.49 (item 11) (all $\mathrm{p}<0.001)$ (Table 3).

The overall test-retest correlation was 0.91 ; and the test-retest correlations for interpersonal and intrapersonal functions were 0.84 and 0.92 , respectively (all $\mathrm{p}<0.001$ ).

\section{Validity analysis}

\section{Exploratory Factor Analysis (EFA)}

Inspection of the scree plot and eigenvalues indicated a 2 -factor structure accounting for $40.57 \%$ of the total variance. Regarding the 2 -factor structure, factor 1 had an eigenvalue of 13.01 and included interpersonal functions. Factor 2 had an eigenvalue of 2.76 and included intrapersonal functions. The intercorrelation between the 2 factors was adequate $(\mathrm{r}=0.58, \mathrm{p}=0.05)$. Thirty-four out of 39 items loaded on the interpersonal or intrapersonal functions consistent with previous studies. ${ }^{11,17}$ Two items (item 7 and 17) loaded on intrapersonal functions rather than interpersonal functions, and three items (item 11, 31 and 32) loaded on interpersonal functions rather than intrapersonal functions (Figure 1, Supplementary Material 2 in the online-only Data Supplement).

\section{Confirmatory Factor Analysis (CFA)}

The current analysis defined the following 13 observed variables and 2 implicit factors of the Korean version of the ISAS functional scales: ${ }^{12,17}$ intrapersonal functions (affect regulation, avoiding dissociation, avoiding suicide, marking distress and self-punishment) and interpersonal functions (autonomy, 
interpersonal influence, interpersonal boundaries, peer bonding, revenge, self-care, sensation seeking and toughness) (Figure 2). In the CFA, the measurement model showed acceptable validity $\left(\chi^{2}=423.45\right.$, degrees of freedom $=64$, RMSEA $=$
$0.10, \mathrm{CFI}=0.91, \mathrm{RMR}=0.01, \mathrm{n}=539)$. The factor loadings varied from 0.42 (sensation seeking) to 2.89 (interpersonal influence), with a median of 1.75 (revenge).

The Pearson correlation for the ISAS functional scales and

Table 3. Correlations between the overall score and each item in the ISAS section $2(N=539)$

\begin{tabular}{|c|c|}
\hline Each function (number) & ISAS section 2 overall \\
\hline 01. When I self-harm, I am calming myself down. & $0.29^{*}$ \\
\hline 02. When I self-harm, I am creating a boundary between myself and others. & $0.65^{*}$ \\
\hline 03. When I self-harm, I am punishing myself. & $0.55^{*}$ \\
\hline 04. When I self-harm, I am giving myself a way to care for myself (by attending to the wound). & $0.44^{*}$ \\
\hline 05. When I self-harm, I am causing pain so I will stop feeling numb. & $0.45^{*}$ \\
\hline 06. When I self-harm, I am avoiding the impulse to attempt suicide. & $0.55^{*}$ \\
\hline 07. When I self-harm, I am doing something to generate excitement or exhilaration. & $0.39^{*}$ \\
\hline 08. When I self-harm, I am bonding with peers. & $0.30^{*}$ \\
\hline 09. When I self-harm, I am letting others know the extent of my emotional pain. & $0.41^{*}$ \\
\hline 10. When I self-harm, I am seeing if I can stand the pain. & $0.48^{*}$ \\
\hline 11. When I self-harm, I am creating a physical sign that I feel awful. & $0.49^{*}$ \\
\hline 12. When I self-harm, I am getting back at someone. & $0.38^{*}$ \\
\hline 13. When I self-harm, I am ensuring that I am self-sufficient. & $0.38^{*}$ \\
\hline 14. When I self-harm, I am releasing emotional pressure that has built up inside of me. & $0.50^{*}$ \\
\hline 15. When I self-harm, I am demonstrating that I am separate from other people. & $0.66^{*}$ \\
\hline 16. When I self-harm, I am expressing anger towards myself for being worthless or stupid. & $0.62^{*}$ \\
\hline 17. When I self-harm, I am creating a physical injury that is easier to care for than my emotional distress. & $0.66^{*}$ \\
\hline 18. When I self-harm, I am trying to feel something (as opposed to nothing) even if it is physical pain. & $0.62^{*}$ \\
\hline 19. When I self-harm, I am responding to suicidal thoughts without actually attempting suicide. & $0.61^{*}$ \\
\hline 20. When I self-harm, I am entertaining myself or others by doing something extreme. & $0.43^{*}$ \\
\hline 21. When I self-harm, I am fitting in with others. & $0.34^{*}$ \\
\hline 22. When I self-harm, I am seeking care of help from others. & $0.50^{*}$ \\
\hline 23. When I self-harm, I am demonstrating I am tough or strong. & $0.48^{*}$ \\
\hline 24. When I self-harm, I am proving to myself that my emotional pain is real. & $0.68^{*}$ \\
\hline 25. When I self-harm, I am getting revenge against others. & $0.49^{*}$ \\
\hline 26. When I self-harm, I am demonstrating that I do not need to rely on others for help. & $0.49^{*}$ \\
\hline 27. When I self-harm, I am reducing anxiety, frustration, anger, or other overwhelming emotions. & $0.51^{*}$ \\
\hline 28. When I self-harm, I am establishing a barrier between myself and others. & $0.62^{*}$ \\
\hline 29. When I self-harm, I am reacting to feeling unhappy with myself or disgusted with myself. & $0.71^{*}$ \\
\hline 30. When I self-harm, I am allowing myself to focus on treating the injury, which can be gratifying or satisfying. & $0.29^{*}$ \\
\hline 31. When I self-harm, I am making sure I am still alive when I don't feel real. & $0.57^{*}$ \\
\hline 32. When I self-harm, I am putting a stop to suicidal thoughts. & $0.62^{*}$ \\
\hline 33. When I self-harm, I am pushing my limits in a manner akin to skydiving or other extreme activities. & $0.37^{*}$ \\
\hline 34. When I self-harm, I am creating a sign of friendship or kinship with friends of loved ones. & $0.24^{*}$ \\
\hline 35. When I self-harm, I am keeping a loved one from leaving or abandoning me. & $0.51^{*}$ \\
\hline 36. When I self-harm, I am proving I can take the physical pain, & $0.48^{*}$ \\
\hline 37. When I self-harm, I am signifying the emotional distress I', experiencing. & $0.68^{*}$ \\
\hline 38. When I self-harm, I am trying to hurt someone close to me. & $0.47^{*}$ \\
\hline 39. When I self-harm, I am establishing that I am autonomous/independent. & $0.59^{*}$ \\
\hline
\end{tabular}

${ }^{*} \mathrm{p}<0.05$. ISAS: Inventory of Statements about Self-Injury 


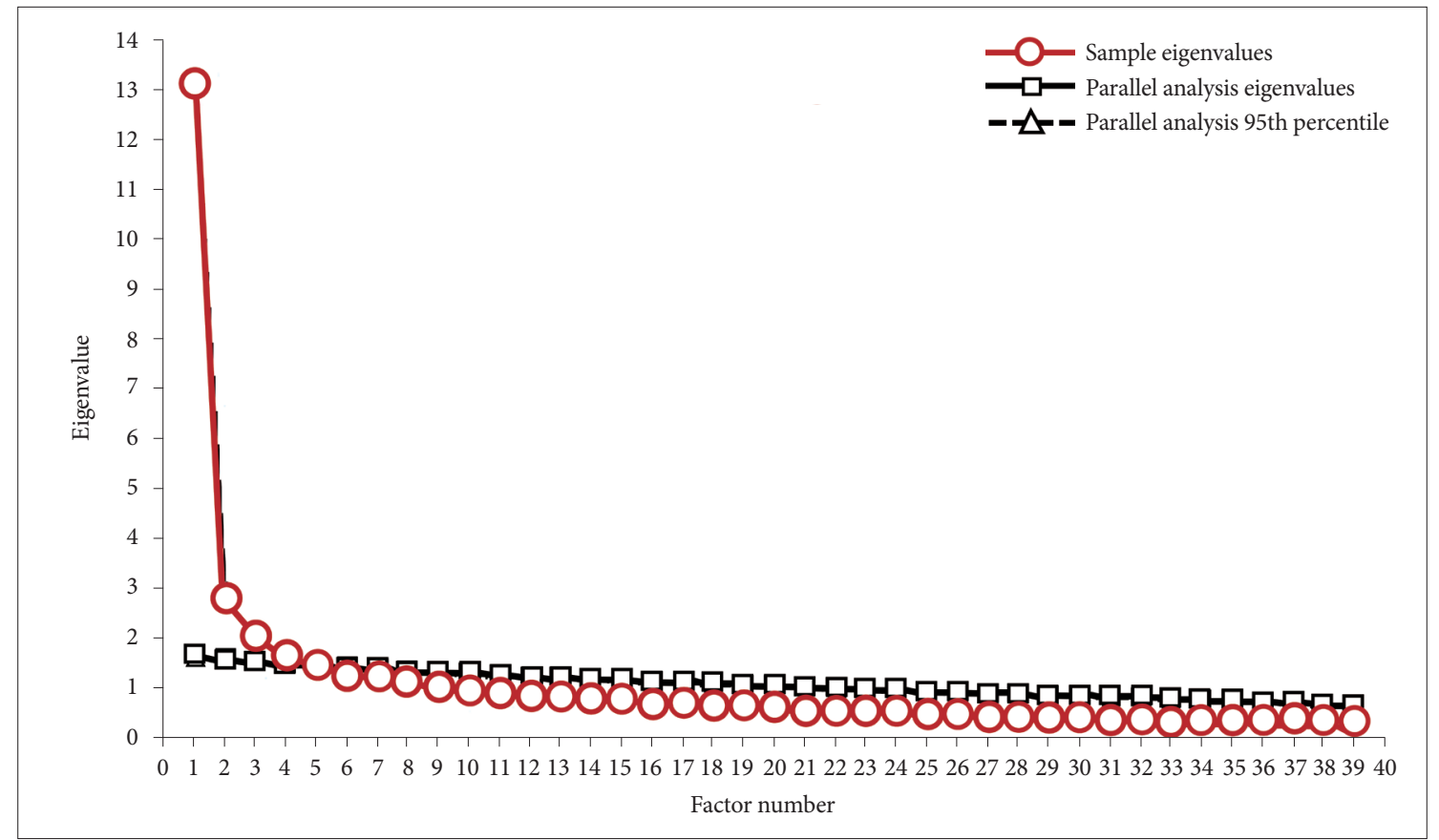

Figure 1. Scree plot for the exploratory factor analysis of the Inventory of Statements About Self-injury.

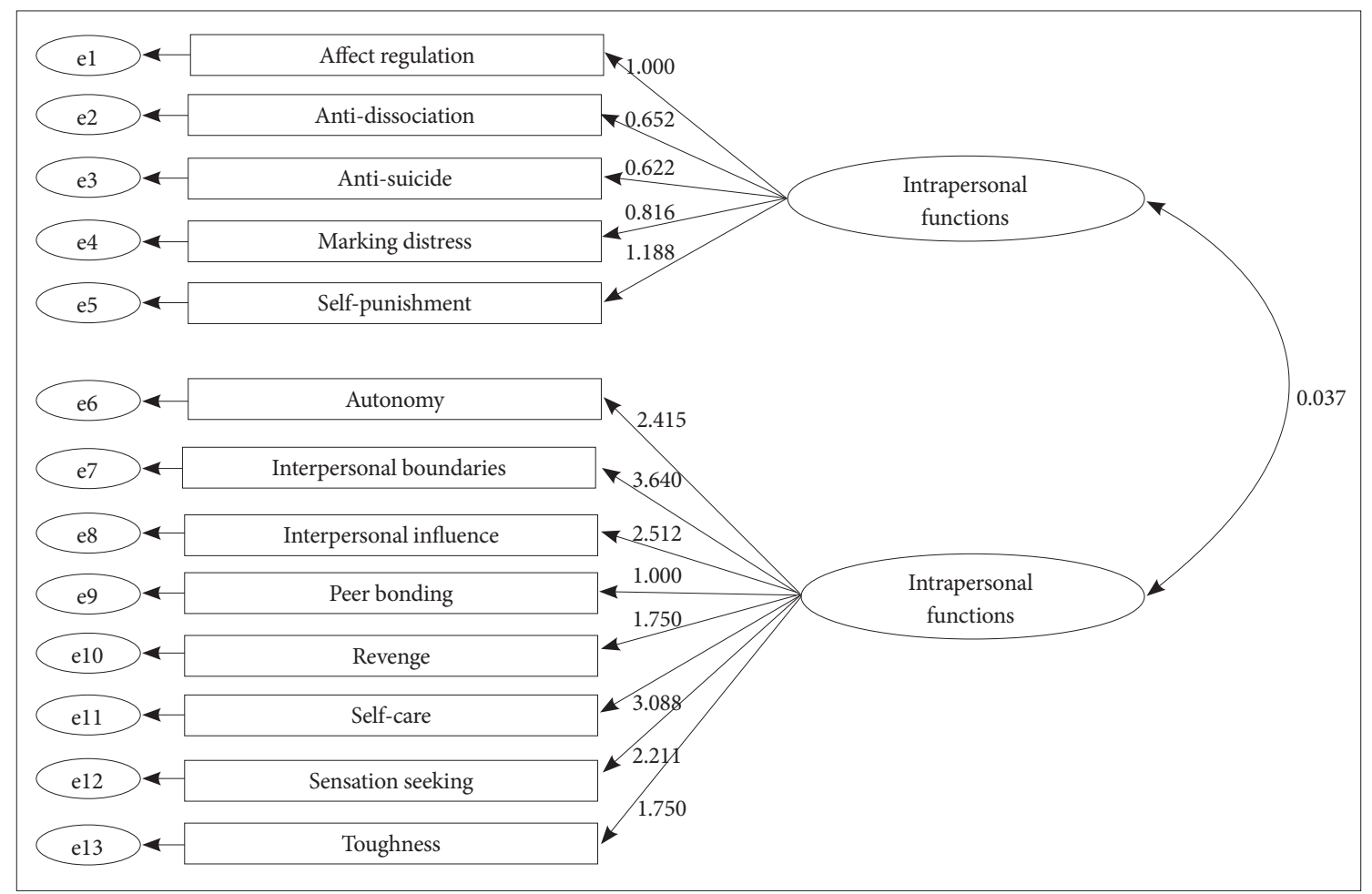

Figure 2. The structure of the Inventory of Statements About Self-injury functions.

NSSI functions assessed by the FASM (item "H") was calculated to investigate concurrent validity. The correlation between the overall score of ISAS section 2 and the FASM was good $(\mathrm{r}=0.77, \mathrm{p}<0.001$; intrapersonal function: $\mathrm{r}=0.75, \mathrm{p}<0.001$; interpersonal function: $r=0.70, \mathrm{p}<0.001$ ).

\section{Relationship to other clinical measures}

Correlations between the Korean version of the ISAS and other clinical scales were analyzed to better understand the psychopathological characteristics related to NSSI. Regarding the NSSI behaviors (ISAS section 1), the frequency of NSSI 
Table 4. Correlations between the ISAS and other scales

\begin{tabular}{lcccc}
\hline & $\begin{array}{c}\text { ISAS } \\
\text { section 1 }\end{array}$ & $\begin{array}{c}\text { ISAS } \\
\text { section 2 }\end{array}$ & $\begin{array}{c}\text { Intrapersonal } \\
\text { functions }\end{array}$ & $\begin{array}{c}\text { Interpersonal } \\
\text { functions }\end{array}$ \\
\hline BSI & $0.48^{*}$ & $0.57^{*}$ & $0.62^{*}$ & $0.17^{*}$ \\
KRQ-30 & $-0.33^{*}-$ & $-0.43^{*}$ & $-0.37^{*}$ & $-0.47^{*}$ \\
\hline
\end{tabular}

${ }^{*} \mathrm{p}<0.05$. ISAS: Inventory of Statements about Self-Injury, ISAS section 1: NSSI behaviors, ISAS section 2: NSSI functions, BSI: Beck Scale for Suicidal Intention, KRQ-53: Korean Resilience Questionnaire-53

behavior showed a positive correlation with suicidal ideation $(\mathrm{r}=0.48, \mathrm{p}<0.001)$ and a negative correlation with resilience $(\mathrm{r}=$ $-0.33, \mathrm{p}<0.001)$. More specifically, intrapersonal function was related to high suicidal ideation $(\mathrm{r}=0.62, \mathrm{p}<0.001)$ and low resilience $(\mathrm{r}=-0.45, \mathrm{p}<0.001)$. Additionally, interpersonal function was related to high suicidal ideation $(\mathrm{r}=0.47, \mathrm{p}<0.001)$ and low resilience $(\mathrm{r}=-0.38, \mathrm{p}<0.001)$ (Table 4$)$.

\section{DISCUSSION}

Just a decade ago, self-injury was regarded as a dramatic gesture by immature individuals who would seek the attention of others or fail to regulate their own emotions. ${ }^{46}$ At present, NSSI is not simply an attempt to attract attention or suicide, but a consequence of a variety of individual-specific functions. ${ }^{11,14}$

The main purpose of this study was to examine the psychometric properties of the Korean version of the ISAS. ${ }^{17}$ As a result, we confirmed the adequate reliability and validity of both the behavioral and functional scales of the ISAS and identified a factor structure of intrapersonal and interpersonal functions for NSSI behaviors, and these findings were similar to the results of previous studies ${ }^{12,17}$ and verified by both EFA and a CFA. We have also shown the relationships between NSSI functions and other psychological vulnerabilities, such as suicidal ideation and poor resilience.

The results of this study are summarized as follows. First, the findings support the good reliability and validity of the Korean version of the ISAS behavioral scales, which were originally designed to investigate the type and lifetime frequency of 12 NSSI behaviors. The statistical properties of the Korean version of the ISAS behavioral sections were similar to those found in the original study ${ }^{17}$ and in the Turkish study. ${ }^{12}$ For instance, in terms of internal consistency, every self-injurious behavior pattern was significantly correlated with the total frequency of NSSI measured by the Korean version of the ISAS. ${ }^{12}$ However, compared with other NSSI behaviors, such as cutting, biting, carving, burning, and swallowing, the use of dangerous substances showed weakened relationships with both the ISAS and the FASM frequency scales. These findings are in accordance with previous research ${ }^{47}$ Therefore, given the high variance in the frequency of various NSSI types, the search for specific self-injurious behavior domains should be prioritized above the other types of the wide range of NSSI behaviors.

Regarding the second section of the ISAS, we confirmed the sufficient reliability and validity, which is consistent with the findings of the original, Turkish, and Mexican studies. ${ }^{12,17,23}$ Moreover, we replicated a two-factor structure of NSSI functions, which is consistent with previous research across various ages and cultures. ${ }^{15,22,30,48,49}$ More specifically, the intrapersonal functions of NSSI among Korean adults included affect regulation, avoiding dissociation, avoiding suicide, marking distress, and self-punishment. Interpersonal functions are related to autonomy, interpersonal boundaries, interpersonal influence, peer bonding, revenge, self-care, sensation seeking, and toughness. Additionally, as with previous research, the fit indices were acceptable, indicating good construct validity. For example, the RMSEA was slightly higher than the conventional acceptance limits but still showed mediocre fit $\left(0.08^{-}\right.$ $0.10){ }^{43}$ This finding was also consistent with the results of the Turkish study (RMSEA $=0.08){ }^{12}$

The results of the correlation analysis revealed that the selfreported NSSI functions measured in the Korean version of the ISAS were significantly correlated with problematic clinical features, such as suicidal ideation and reduced resilience. In other words, the participants who exhibited more suicidal ideation and less resilience reported more functions for their self-injurious behaviors. As previous research has illustrated, vulnerable individuals may initiate NSSI when they have difficulty coping with internal and external environments. ${ }^{12} \mathrm{Re}-$ silience, one of the most invaluable psychological resources, reduces the impact of stressful life events on individuals ${ }^{50}$ and attenuates the likelihood of suicide among adolescents and young adults. ${ }^{51}$ Indeed, a low level of resilience is the only significant predictor of current NSSI behaviors according to Rotolone and Martin. ${ }^{26}$ Therefore, further study is needed to confirm the dynamics between NSSI, suicidal ideation, and resilience in order to seek effective interventions to prevent the lethal consequences of self-harming behaviors.

Certain limitations were observed in this study. First, this study was conducted with a convenient sample of individuals who could access the internet, and participation in research was available only through the internet. Most of the participants were university students living in the capital area, which prevents the generalization of the findings to other populations. Second, although the validation analyses used are widely accepted as robust research methodologies, the findings were derived from limited self-reported data. Therefore, to thoroughly identify antecedents and precedents for NSSI in Korea, further research should include different samples and methodologies. Because NSSI can lead to later suicidal behav- 
iors, historical suicide attempts should be included in future studies.

In conclusion, the Korean version of the ISAS, which measures the frequency and function of NSSI, is a reliable and valid measure of the NSSI behaviors in Korean adults. The Korean version of the ISAS functional scales also has a 2-factor structure that includes intrapersonal and interpersonal functions, which is consistent with previous studies. The use of various psychological measures extends the research area of mental health. We hope that a more sophisticated approach will become available for both a clinical setting and research setting based on the results presented here on the relationships among NSSI, suicidal ideation and weakened resilience as well as the adequate psychometric properties of the Korean version of the ISAS.

\section{Supplementary Materials}

The online-only Data Supplement is available with this article at https://doi.org/10.30773/pi.2019.01.23.

\section{Acknowledgments}

This work was supported by the National Research Foundation of Korea (NRF) by a grant funded by the Korean government (NRF-2017R1C1B50 18400), and by the Chung-Ang University Research Grants in 2018.

\section{Conflicts of Interest}

The authors have no potential conflicts of interest to disclose.

\section{REFERENCES}

1. Liu RT, Cheek SM, Nestor BA. Non-suicidal self-injury and life stress: a systematic meta-analysis and theoretical elaboration. Clin Psychol Rev 2016;47:1-14.

2. Jacobson CM, Gould M. The epidemiology and phenomenology of non-suicidal self-injurious behavior among adolescents: a critical review of the literature. Arch Suicide Res 2007;11:129-147.

3. Nock MK, Park JM, Finn CT, Deliberto TL, Dour HJ, Banaji MR. Measuring the suicidal mind: implicit cognition predicts suicidal behavior. Psychol Sci 2010;21:511-517.

4. Heath N, Toste J, Nedecheva T, Charlebois A. An examination of nonsuicidal self-injury among college students. J Ment Health Couns 2008; 30:137-156.

5. Jacobson CM, Gould M. The epidemiology and phenomenology of non-suicidal self-injurious behavior among adolescents: a critical review of the literature. Arch Suicide Res 2007;11:129-147.

6. Lloyd-Richardson EE, Perrine N, Dierker L, Kelley ML. Characteristics and functions of non-suicidal self-injury in a community sample of adolescents. Psychol Med 2007;37:1183-1192.

7. Heath NL, Ross S, Toste JR, Charlebois A, Nedecheva T. Retrospective analysis of social factors and nonsuicidal self-injury among young adults. Can J Behav Sci 2009;41:180-186.

8. Lee DG, Ham KA, Bae BH. Self-injurious behaviors: suicidal self-injury and non-suicidal self-injury in female middle school students. Korean J Counsel Psychother 2016;28:1171-1192.

9. Ribeiro JD, Franklin JC, Fox KR, Bentley KH, Kleiman EM, Chang BP, et al. Self-injurious thoughts and behaviors as risk factors for future suicide ideation, attempts, and death: a meta-analysis of longitudinal studies. Psychol Med 2015;46:225-236.

10. Guan K, Fox KR, Prinstein MJ. Nonsuicidal self-injury as a time-invari- ant predictor of adolescent suicide ideation and attempts in a diverse community sample. J Consult Clin Psychol 2012;80:842-849.

11. Klonsky ED, Glenn CR, Styer DM, Olino TM, Washburn JJ. The functions of nonsuicidal self-injury: converging evidence for a two-factor structure. Child Adolesc Psychiatry Ment Health 2015;9:44.

12. Bildik T, Somer O, Kabukçu Başay B, Başay Ö, Özbaran B. The validity and reliability of the Turkish version of the inventory of statements about self-injury. Turk Psikiyatri Dergisi 2013;24:49-57.

13. Nock MK, Prinstein MJ. A functional approach to the assessment of self-mutilative behavior. J Consult Clin Psychol 2004;72:885-890.

14. Nock MK, Prinstein MJ, Sterba SK. Revealing the form and function of self-injurious thoughts and behaviors: a real-time ecological assessment study among adolescents and young adults. J Abnorm Psychol 2009;118:816-827.

15. Taylor PJ, Jomar K, Dhingra K, Forrester R, Shahmalak U, Dickson JM. A meta-analysis of the prevalence of different functions of nonsuicidal self-injury. J Affect Disord 2018;227:759-769.

16. Kwon HJ. Exploration of Eotional and Cognitive Factors Influencing Non-suicidal Self-injury. Seoul: Seoul National University; 2014.

17. Klonsky ED, Glenn CR. Assessing the functions of non-suicidal selfinjury: Psychometric properties of the Inventory of Statements About Self-injury (ISAS). J Psychopathol Behav Assess 2009;31:215-219.

18. Gratz KL. Measurement of deliberate self-harm: preliminary data on the Deliberate Self-Harm Inventory. J Psychopathol Behav Assess 2001; 23:253-263.

19. Linehan MM, Comtois KA, Brown MZ, Heard HL, Wagner A. Suicide Attempt Self-Injury Interview (SASII): development, reliability, and validity of a scale to assess suicide attempts and intentional self-injury. Psychol Assess 2006;18:303-312.

20. Lloyd E, Kelley M, Hope T. Self-mutilation in a community sample of adolescents: descriptive characteristics and provisional prevalence rates. New Orleans, LA: Annual meeting of the Society for Behavioral Medicine; 1997.

21. Klonsky ED. The functions of deliberate self-injury: a review of the evidence. Clin Psychol Rev 2007;27:226-239.

22. Klonsky ED, Olino TM. Identifying clinically distinct subgroups of self-injurers among young adults: a latent class analysis. J Consult Clin Psychol 2008;76:22-27.

23. Castro Silva E, Benjet C, Juárez García F, Jurado Cárdenas S, Lucio Gómez-Maqueo ME, Valencia Cruz A. Adaptation and psychometric properties of the Inventory of Statements About Self-injury in Mexican students. Acta Investig Psicol 2016;6:2544-2551.

24. Lindholm T, Bjärehed J, Lundh LG. Functions of nonsuicidal self-injury among young women in residential care: a pilot study with the Swedish version of the Inventory of Statements about Self-Injury. Cogn Behav Ther 2011;40:183-189.

25. Kovacs M, Garrison B. Hopelessness and eventual suicide: a 10-year prospective study of patients hospitalized with suicidal ideation. Am J Psychiatry 1985;1:559-563.

26. Rotolone C, Martin G. Giving up self-Injury: a comparison of everyday social and personal resources in past versus current self-injurers. Arch Suicide Res 2012;16:147-158.

27. Glenn CR, Klonsky ED. Prospective prediction of nonsuicidal self-injury: a 1-year longitudinal study in young adults. Behav Ther 2011;42: 751-762.

28. Abramson LY, Metalsky GI, Alloy LB. Hopelessness depression: a theory-based subtype of depression. Psychol Rev 1989;96:358-372.

29. Eysenck HJ. Manual of the Eysenck Personality Questionnaire. London: Hodder \& Stoughton; 1975.

30. Kwon HJ, Kwon SM. Validation study of the Korean version of the Functional Assessment of Self-Mutilation (FASM). Clin Psychol Kor Res Pract 2017;3:187-205.

31. Richard WB. Back-translation for cross-cultural research. J Cross Cult Psychol 1970;1:185-216.

32. Kinzie JD, Manson SM. The use of self-rating scales in cross-cultural 
psychiatry. Hosp Community Psychiatry 1987;38:190-196.

33. Beck AT, Kovacs M, Weissman A. Assessment of suicidal intention: the Scale for Suicide Ideation. J Consult Clin Psychol 1979;47:343-352.

34. Holi MM, Pelkonen M, Karlsson L, Kiviruusu O, Ruuttu T, Heilä H, et al. Psychometric properties and clinical utility of the Scale for Suicidal Ideation (SSI) in adolescents. BMC Psychiatry 2005;5:8.

35. Shin M, Park K, Oh K, Kim Z. Study of Suicidal Ideation among High School Students. Korean J Clin Psychol 1990;9:1-19.

36. Tusaie K, Dyer J. Resilience: a historical review of the construct. Holist Nurs Pract 2004;18:3-10.

37. Reivich K, Shatte A. The Resilience Factor: 7 Keys to Finding Your Inner Strength and Overcoming Life's Hurdles. New York: Harmony; 2003.

38. Huh K, Choi WJ, Seok JH. Relationship of suicide risk with early life stress and resilience in patients with major depressive disorder. J Korean Neuropsychiatr Assoc 2014;53:386-393.

39. Browne MW. An overview of analytic rotation in exploratory factor analysis. Multivar Behav Res 2001;36:111-150.

40. Hu Lt, Bentler PM. Cutoff criteria for fit indexes in covariance structure analysis: conventional criteria versus new alternatives. Struct Equ Modeling 1999;6:1-55.

41. Browne MW, Cudeck R. Alternative Ways of Assessing Model Fit. Newbury Park, CA: Sage; 1993.

42. MacCallum RC, Browne MW, Sugawara HM. Power analysis and determination of sample size for covariance structure modeling. Psychol Methods 1996;1:130-149.

43. Byrne B. Structural Equation Modeling with AMOS Basic Concepts,
Applications and Programming (2nd Ed.). New York: Taylor \& Francis Group; 2010.

44. Marsh HW, Hau KT, Wen Z. In search of golden rules: comment on hypothesis-testing approaches to setting cutoff values for fit indexes and dangers in overgeneralizing Hu and Bentler's (1999) findings. Struct Equ Modeling 2004;11:320-341.

45. Brown TA. Confirmatory Factor Analysis for Applied Research. New York: Guilford Publications; 2014.

46. Victor SE, Davis T, Klonsky ED. Descriptive characteristics and initial psychometric properties of the non-suicidal self-injury disorder scale. Arch Suicide Res 2017;21:265-278.

47. Catherine RG, Klonsky ED. One-year test-retest reliability of the Inventory of Statements about Self-Injury (ISAS). Assessment 2011;18:375378.

48. Turner BJ, Chapman AL, Layden BK. Intrapersonal and interpersonal functions of non suicidal self-injury: associations with emotional and social functioning. Suicide Life Threat Behav 2012;42:36-55.

49. Wang B, You J, Lin MP, Xu S, Leung F. Developmental trajectories of nonsuicidal self-injury in adolescence and intrapersonal/ interpersonal risk factors. J Res Adolesc 2017;27:392-406.

50. Johnson J, Wood AM, Gooding P, Taylor PJ, Tarrier N. Resilience to suicidality: the buffering hypothesis. Clin Psychol Rev 2011;31:563-591.

51. Nrugham L, Holen A, Sund AM. Associations between attempted suicide, violent life events, depressive symptoms, and resilience in adolescents and young adults. J Nerv Ment Dis 2010;198:131-136. 